\title{
Thermal characterisation of quantum cascade lasers with Fabry Perot modes
}

\author{
Sinan Gündoğdu ${ }^{\mathrm{a}}$, Hadi Sedaghat Pisheh ${ }^{\mathrm{b}}$, Abdullah Demirc $^{\mathrm{c}}$, Mete Günoven ${ }^{\mathrm{d}}$, Atilla Aydınlı ${ }^{*}$, \\ and Carlo Sirtorif \\ aPhysics Department, Bilkent University, Ankara 06800, Turkey \\ bMechanical Engineering Department, Bilkent University, Ankara 06800, Turkey \\ ${ }^{\mathrm{c} N a t i o n a l ~ N a n o t e c h n o l o g y ~ R e s e a r c h ~ C e n t e r, ~ B i l k e n t ~ U n i v e r s i t y, ~ A n k a r a ~ 06800, ~ T u r k e y ~}$ \\ ${ }^{d}$ Physics Department, Middle East Technical University, Ankara 06800, Turkey \\ eElectrical and Electronics Engineering Department, Uludag University, Bursa, 16059 Turkey \\ fLaboratoire Matériaux et Phénomènes Quantiques, Université Paris Diderot-Paris7, Paris, \\ France
}

\begin{abstract}
Quantum cascade lasers are coherent light sources that rely on intrersubband transition in periodic semiconductor quantum well structures. They operate at frequencies from mid-infrared to terahertz. In cases of long wavelength and typical low thermal conductivity of the active region, temperature rise in the active region during operation is a major concern. Thermal conductivity of QCL epi-layers differ significantly from the values of bulk semiconductors and measurement of the thermal conductivity of epi-layers is critical for design. It is well known that Fabry-Perot spectra of QCL cavities exhibit fine amplitude oscillations with frequency and can be used for real time in-situ temperature measurement. Phase of the modulation depends on the group refractive index of the cavity, which depends on the cavity temperature. We fabricated QCL devices with from 12, to 24 um mesa widths and $2 \mathrm{~mm}$ cavity length and and measured high resolution, high speed time resolved spectra using a FTIR spectrometer in step scan mode in a liquid nitrogen cooled, temperature controlled dewar. We used the time resolved spectra of QCLs to measure average temperature of the active region of the laser as a function of time. We examined the effect of pulse width and duty cycle on laser heating. We measured the temperature derivative of group refractive index of the cavity. Building a numerical model, we estimated the thermal conductivity of active region and calculated the heating of the QCL active region in pulsed mode for various waveguide widths.
\end{abstract}

Keywords: Quantum Cascade Lasers, Thermal Conductivity, Temperature

\section{INTRODUCTION}

Quantum cascade lasers (QCLs) are semiconductor devices that can be tailored to emit light from infrared to terahertz frequencies. They rely on intersubband transitions of multi-quantum wells formed by many layers of semiconductors, hence they can lase at photon energies less than the band-gap of the materials. Since their first demonstration in 1994, ${ }^{1}$ extensive efforts has been made to increase efficiency, high temperature operation and high power output. ${ }^{2}$ Since their relatively thick active region formed by many layers of alternating semiconductors, their thermal behavior is different than other semiconductor lasers like diode lasers. Thermal conductivity of active region is less than the bulk materials constituting it and strongly anisotropic. ${ }^{3}$ Since the energy difference between the intersubband levels is low, they can be easily excited with thermal energy of the lattice. Therefore their operation depends on temperature strongly. For this reason, thermal management of QCLs is an important factor for the design of a QCL and its packaging. ${ }^{4}$

In-situ temperature measurement of a QCL active region yields valuable information about the thermal performance and heat dissipation of the laser. Some methods that has been used to measure temperature of QCLs include microprobe photoluminescence (PL), ${ }^{3,5-7}$ and thermoreflectance. ${ }^{8-11}$ Photoluminescence peak wavelength of the semiconductors depends on temperature. For PL method, slight wavelength shift of the peak is measured by focusing an excitation laser on the facet and collecting the emitted PL light and analyzing the light with a spectrometer. By scanning the focused laser over the facet, a 2D map of temperature can be obtained.

Semiconductor Lasers and Laser Dynamics VIII, edited by Krassimir Panajotov, Marc Sciamanna, Rainer Michalzik, Proc. of SPIE Vol. 10682, 106820J · @ 2018 SPIE · CCC code: 0277-786X/18/\$18 · doi: 10.1117/12.2311651 
Signal Intensity (a.u.)

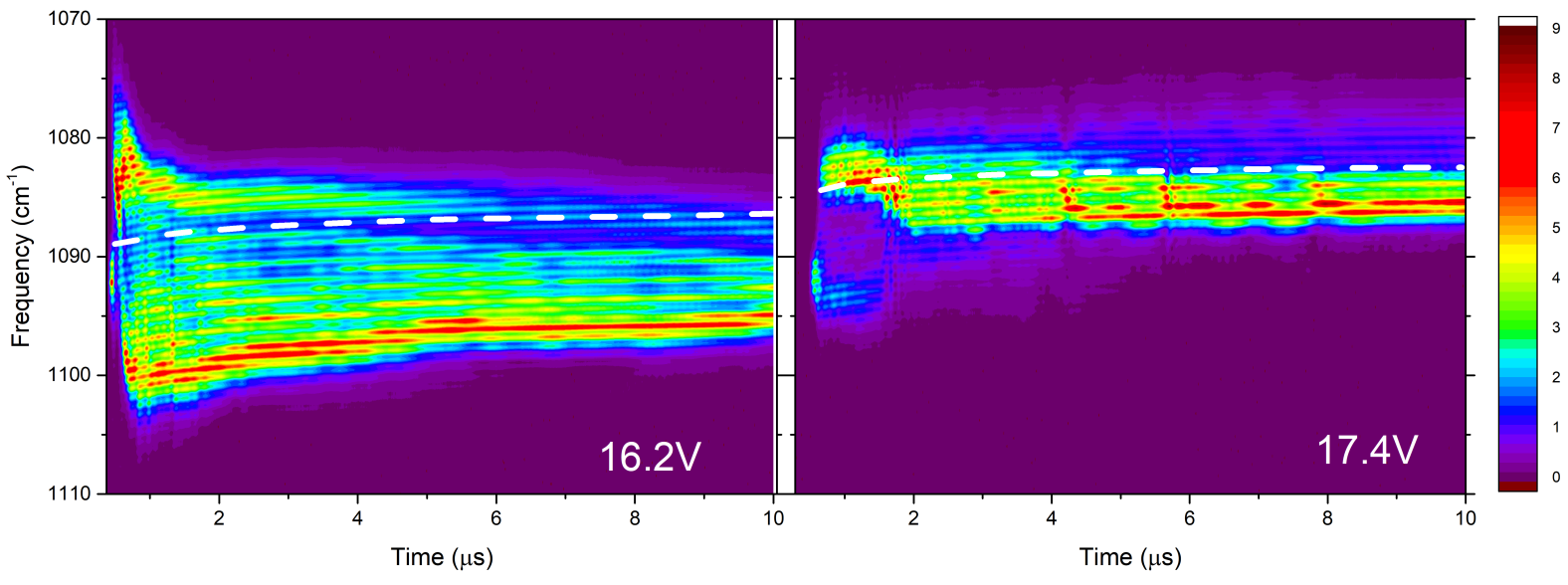




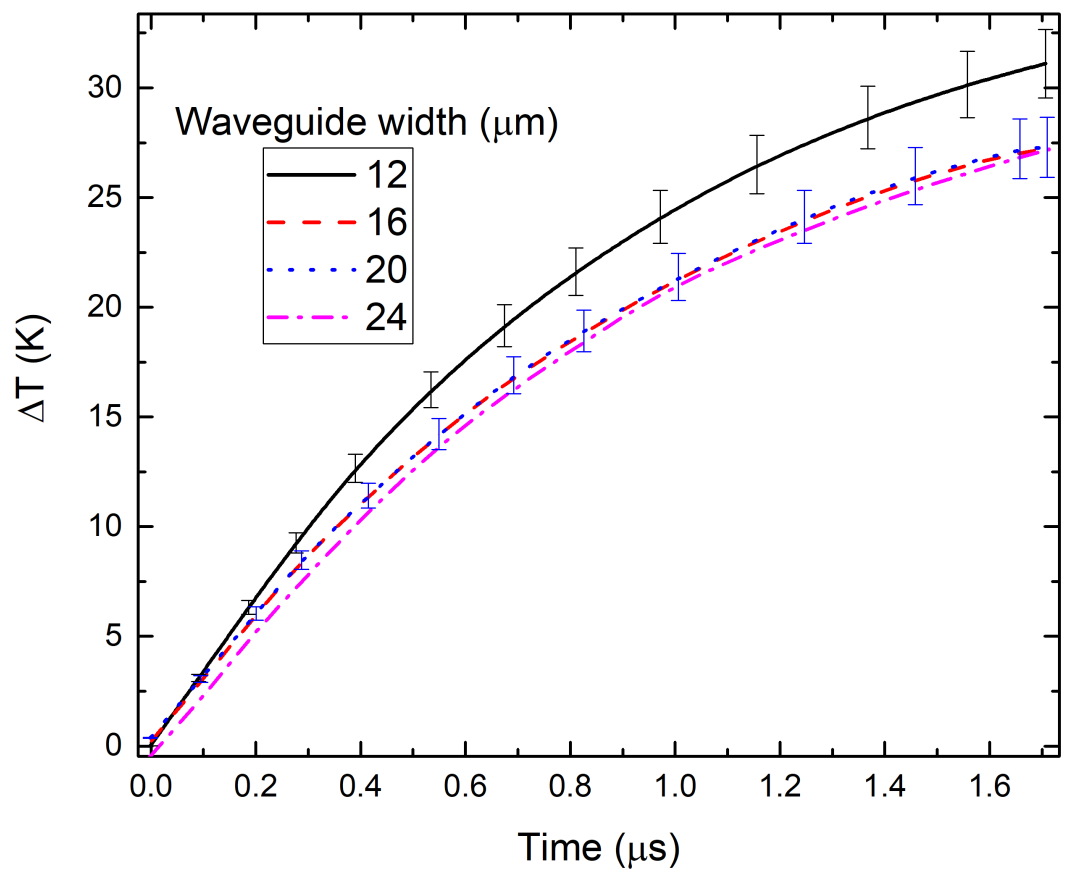

Proc. of SPIE Vol. 10682 106820J-3 


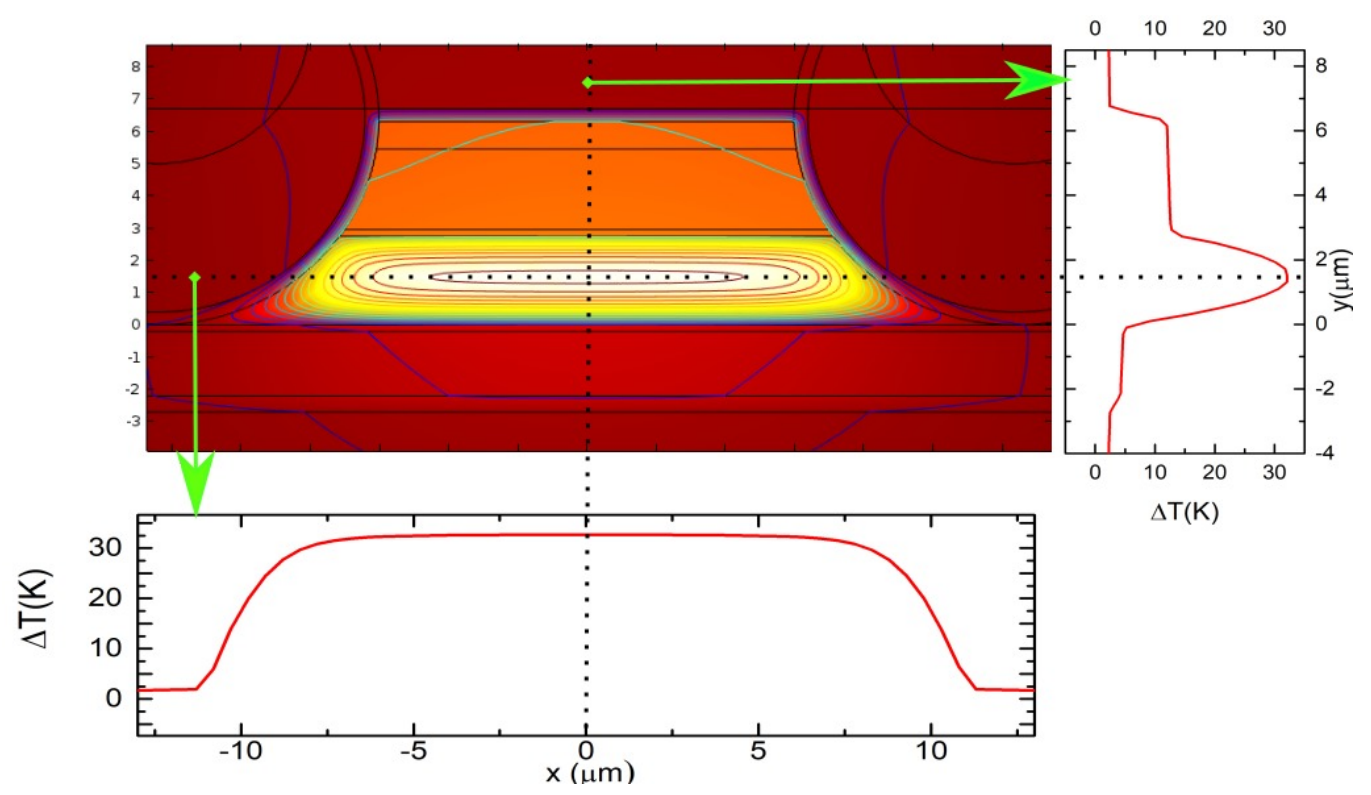

Proc. of SPIE Vol. 10682 106820J-4 


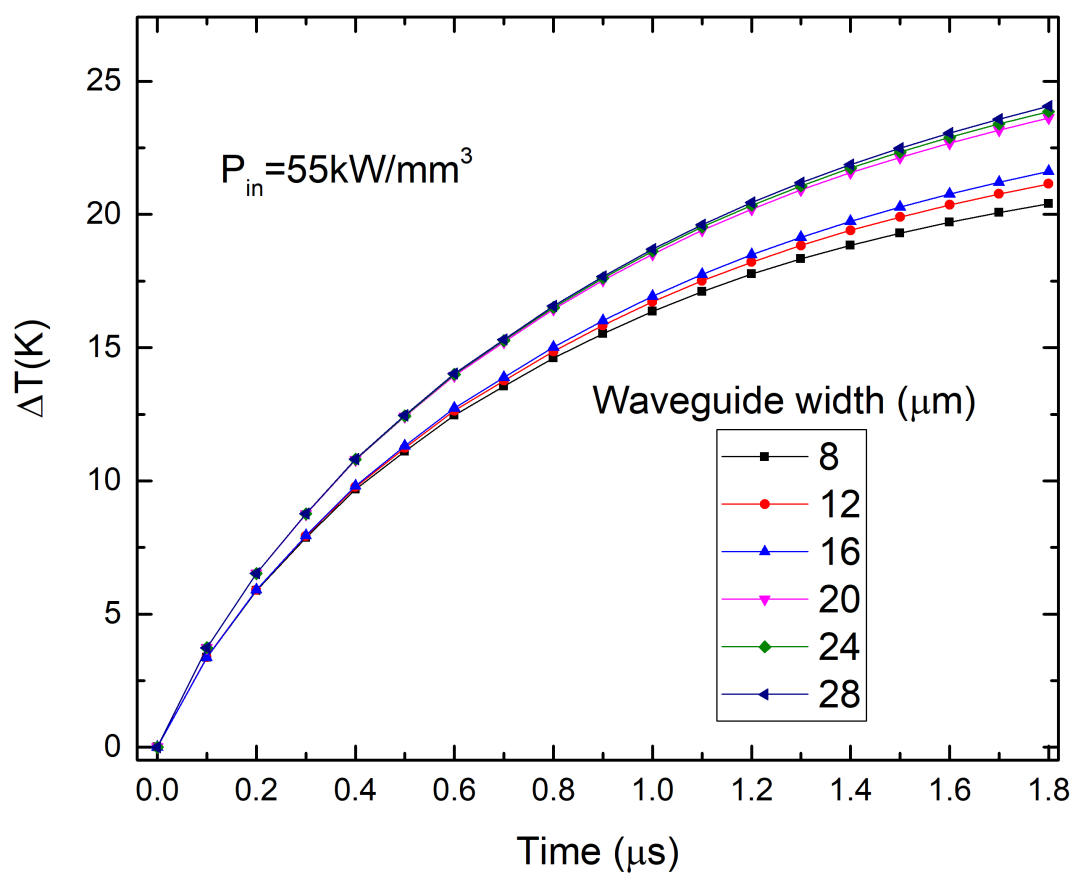

Proc. of SPIE Vol. 10682 106820J-5 
[3] Vitiello, M. S. and Scamarcio, G., "Anisotropic heat propagation velocity in quantum cascade lasers," Applied Physics Letters 96, 101101 (mar 2010).

[4] Spagnolo, V., Lops, A., Scamarcio, G., Vitiello, M. S., and Franco, C. D., "Improved thermal management of mid-IR quantum cascade lasers," Journal of Applied Physics 103, 043103 (feb 2008).

[5] Spagnolo, V., Scamarcio, G., Marano, D., Troccoli, M., Capasso, F., Gmachl, C., Sergent, A., Hutchinson, A., Sivco, D., Cho, A., Page, H., Becker, C., and Sirtori, C., "Thermal characteristics of quantum-cascade lasers by micro-probe optical spectroscopy," IEE Proceedings - Optoelectronics 150(4), 298 (2003).

[6] Fathololoumi, S., Ban, D., Luo, H., Dupont, E., Laframboise, S. R., Boucherif, A., and Liu, H. C., "Thermal behavior investigation of terahertz quantum-cascade lasers," IEEE Journal of Quantum Electronics 44, 1139-1144 (dec 2008).

[7] Vitiello, M. S., Scamarcio, G., and Spagnolo, V., "Temperature dependence of thermal conductivity and boundary resistance in THz quantum cascade lasers," IEEE Journal of Selected Topics in Quantum Electronics 14(2), 431-435 (2008).

[8] Pierściński, K., Pierścińska, D., Iwińska, M., Kosiel, K., Szerling, A., Karbownik, P., and Bugajski, M., "Investigation of thermal properties of mid-infrared AlGaAs/GaAs quantum cascade lasers," Journal of Applied Physics 112, 043112 (aug 2012).

[9] Pierścińska, D., Pierściński, K., Płuska, M., Marona, Ł., Wiśniewski, P., Perlin, P., and Bugajski, M., "Examination of thermal properties and degradation of InGaN - based diode lasers by thermoreflectance spectroscopy and focused ion beam etching," AIP Advances 7, 075107 (jul 2017).

[10] Pierścińska, D., Kozlowska, A., Pierściński, K., Bugajski, M., Tomm, J. W., Ziegler, M., and Weik, F., "Thermal processes in high-power laser bars investigated by spatially resolved thermoreflectance," Journal of Materials Science: Materials in Electronics 19, 150-154 (mar 2008).

[11] Pierścińska, D., Pierściński, K., Morawiec, M., Karbownik, P., Gutowski, P., and Bugajski, M., "CCD thermoreflectance spectroscopy as a tool for thermal characterization of quantum cascade lasers," Semiconductor Science and Technology 31, 115006 (sep 2016).

[12] Wang, Q. J., Pflügl, C., Diehl, L., Capasso, F., Edamura, T., Furuta, S., Yamanishi, M., and Kan, H., "High performance quantum cascade lasers based on three-phonon-resonance design," Applied Physics Letters 94, 011103 (jan 2009).

[13] Gundogdu, S., Pisheh, H. S., Demir, A., Gunoven, M., Aydinli, A., and Sirtori, C., "Time resolved fabryperot measurements of cavity temperature in pulsed QCLs," Optics Express 26, 6572 (mar 2018).

[14] Cherroret, N., Chakravarty, A., and Kar, A., "Temperature-dependent refractive index of semiconductors," Journal of Materials Science 43, 1795-1801 (Mar 2008). 